J. Nonlinear Var. Anal. 5 (2021), No. 2, pp. 251-265

Available online at http://jnva.biemdas.com

https://doi.org/10.23952/jnva.5.2021.2.05

\title{
ON BOUNDED SOLUTIONS OF SEMILINEAR FRACTIONAL ORDER DIFFERENTIAL INCLUSIONS IN HILBERT SPACES
}

\author{
M. KAMENSKII ${ }^{1}$, S. KORNEV ${ }^{2}$, V. OBUKHOVSKII ${ }^{2}$, N.C. WONG $^{3, *}$ \\ ${ }^{1}$ Department of Mathematics, Voronezh State University, Voronezh, 394018, Russia \\ ${ }^{2}$ Department of Physics and Mathematics, Voronezh State Pedagogical University, Voronezh, 394043, Russia \\ ${ }^{3}$ Department of Applied Mathematics, National Sun Yat-sen University, Kaohsiung, 80424, Taiwan, and School of \\ Mathematical Sciences, Tiangong University, Tianjin 300387, China
}

\begin{abstract}
We prove a result on a priori estimate for mild solutions to the initial value problem for a semilinear fractional-order differential inclusion in a separable Hilbert space. We assume that the linear part of the inclusion is presented by an unbounded strictly negatively defined operator and the multivalued nonlinearity satisfies an one-sided estimate. To prove this result, we use approximation methods based on, in particular, Yosida approximations of the linear part of the inclusion. The obtained result is applied to justify the existence of mild solutions to the initial value problems on finite intervals, and to prove the existence of mild solutions which are bounded on the semi-axis.

Keywords. Fractional differential inclusion; Semilinear differential inclusion; Cauchy problem; A priori estimate; Bounded solution.
\end{abstract}

\section{INTRODUCTION}

It is well known (see, for example, [1-3]) that, in the case of differential equations, the condition of smoothness of right-hand sides does not provide the existence of solutions to the initial value problem on an unbounded interval. For the existence of such solutions, in the theory of ordinary differential equations, the method of one-sided estimates was widely applied (see, for example, [1]). For a differential equation in a Hilbert space $H$ of the form

$$
x^{\prime}=f(t, x),
$$

one of the simplest estimations of this type can be presented by the inequality

$$
\langle f(t, x), x\rangle \leq a\|x\|^{2}+b .
$$

To obtain the existence of solutions on unbounded intervals in the case of fractional-order equations and inclusions, the condition of the sublinear growth of the right-hand side was used (see [4]-[15]). It is evident that this condition is much stronger than (1.1).

In this paper, we prove the existence of mild solutions of such type for a semilinear fractionalorder differential inclusion in a separable Hilbert space under the assumptions that the linear

\footnotetext{
${ }^{*}$ Corresponding author.

E-mail addresses: mikhailkamenski@mail.ru (M. Kamenskii), kornev_vrn@rambler.ru (S. Kornev), valerioob2000@mail.ru (V. Obukhovskii), wong@math.nsysu.edu.tw (N.-C. Wong).

Received October 18, 2020; Accepted December 29, 2020.
}

(C)2021 Journal of Nonlinear and Variational Analysis 
part of the inclusion is presented by an unbounded strictly negatively defined operator and the multivalued nonlinearity satisfies the estimate of type (1.1). Notice that our construction is essentially based on the following property of the Caputo fractional derivative of a function $x(t)$ in a Hilbert space:

$$
{ }^{C} D_{0}^{q}\|x(t)\|^{2} \leq\left\langle x(t),{ }^{C} D_{0}^{q} x(t)\right\rangle,
$$

which was studied in [16-18].

The paper is organized in the following way. In the next section, we present some preliminaries from the fractional analysis and the theory of condensing multivalued maps. In the third section, we first give a result on a priori estimate for solutions to the initial value problem of a semilinear fractional differential inclusion in a Hilbert space, under the assumption that the linear part of the inclusion is negatively defined and the multivalued nonlinearity satisfies an one-sided estimate. To prove this result, we use approximation methods based on, in particular, the Yosida approximations of the linear part of the inclusion. Finally, we apply this result to prove the existence of a mild solution to our initial value problem on each finite interval and verify the existence of mild solutions, which are bounded on the semi-axis.

\section{PRELIMINARIES}

2.1. Fractional derivative. In this section we will recall some notions and definitions which we will need in the sequel (details can be found in, e.g., [19-22]).

Let $E$ be a real Banach space.

Definition 2.1. The Riemann-Liouville fractional derivative of order $q \in(0,1)$ of a continuous function $g:[0, a] \rightarrow E$ is the function $D_{0}^{q} g$ of the following form:

$$
D_{0}^{q} g(t)=\frac{1}{\Gamma(1-q)} \frac{d}{d t} \int_{0}^{t}(t-s)^{-q} g(s) d s
$$

provided that the right-hand side of this equality is well defined, where $\Gamma$ is the Euler gammafunction

$$
\Gamma(r)=\int_{0}^{\infty} s^{r-1} e^{-s} d s
$$

Definition 2.2. The Caputo fractional derivative of order $q \in(0,1)$ of a continuous function $g:[0, a] \rightarrow E$ is the function ${ }^{C} D_{0}^{q} g$ defined in the following way:

$$
{ }^{C} D_{0}^{q} g(t)=\left(D_{0}^{q}(g(\cdot)-g(0))\right)(t)
$$

provided the right-hand side of this equality is well defined.

Definition 2.3. A function of the form

$$
E_{q, \beta}(z)=\sum_{n=0}^{\infty} \frac{z^{n}}{\Gamma(q n+\beta)}, \quad q, \beta>0, z \in \mathbb{C},
$$

is said to be the Mittag-Leffler function.

The Mittag-Leffler function has the following asymptotic representation as $z \rightarrow \infty$ (see, e.g., [19]): 


$$
E_{q, \beta}(z)= \begin{cases}\frac{1}{q} z^{\frac{1-\beta}{q}} e^{z^{\frac{1}{q}}}-\sum_{n=1}^{N-1} \frac{z^{-n}}{\Gamma(\beta-q n)}+O\left(|z|^{-N}\right), & |\arg z| \leq \frac{1}{2} \pi q, \\ -\sum_{n=1}^{N-1} \frac{z^{-n}}{\Gamma(\beta-q n)}+O\left(|z|^{-N}\right), & |\arg (-z)| \leq\left(1-\frac{1}{2} q\right) \pi .\end{cases}
$$

Denote $E_{q, 1}$ by $E_{q}$. Notice that the second of the above formula implies that, in the case $z=\tau<0$ and $0<q<1$,

$$
E_{q}(\tau) \rightarrow 0 \text { as } \tau \rightarrow-\infty
$$

It follows from the relations (see, e.g., [23]) that

$$
E_{q}(-z)=\int_{0}^{\infty} \xi_{q}(\theta) e^{-z \theta} d \theta \quad \text { and } \quad E_{q, q}(-z)=\int_{0}^{\infty} q \theta \xi_{q}(\theta) e^{-z \theta} d \theta
$$

where

$$
\begin{gathered}
\xi_{q}(\theta)=\frac{1}{q} \theta^{-1-\frac{1}{q}} \Psi_{q}\left(\theta^{-1 / q}\right), \\
\Psi_{q}(\theta)=\frac{1}{\pi} \sum_{n=1}^{\infty}(-1)^{n-1} \theta^{-q n-1} \frac{\Gamma(n q+1)}{n !} \sin (n \pi q), \theta \in \mathbb{R}_{+},
\end{gathered}
$$

that both

$$
E_{q}(\tau)>0 \quad \text { and } \quad E_{q, q}(\tau)>0, \quad \text { for } \tau<0 .
$$

Remark 2.4 (See, e.g., [14]). $\xi_{q}(\theta) \geq 0, \int_{0}^{\infty} \xi_{q}(\theta) d \theta=1$, and $\int_{0}^{\infty} \theta \xi_{q}(\theta) d \theta=\frac{1}{\Gamma(q+1)}$.

Consider a scalar equation of the form

$$
{ }^{C} D_{0}^{q} x(t)=\lambda x(t)+f(t), \quad t \in[0, T]
$$

with the initial condition

$$
x(0)=x_{0}
$$

where $\lambda \in \mathbb{R}$ and $f:[0, T] \rightarrow \mathbb{R}$ is a continuous function. By a solution of this problem, we mean a continuous function $x:[0, T] \rightarrow \mathbb{R}$ satisfying the condition (2.7) whose fractional derivative ${ }^{C} D_{0}^{q} x$ is also continuous and satisfies equation (2.6). It is known (see [20, Example 4.9]) that the unique solution of this equation has the form

$$
x(t)=E_{q}\left(\lambda t^{q}\right) x_{0}+\int_{0}^{t}(t-s)^{q-1} E_{q, q}\left(\lambda(t-s)^{q}\right) f(s) d s .
$$

We will need the following auxiliary assertion, which is an analogue of the known Gronwall lemma on integral inequalities.

Lemma 2.5. Let $z:[0, T] \rightarrow \mathbb{R}$ be a bounded measurable function such that ${ }^{C} D_{0}^{q} z$ is continuous and

$$
{ }^{C} D_{0}^{q} z(t) \leq \lambda z(t)+l(t), \quad t \in[0, T]
$$

where $\lambda \in \mathbb{R}$ and $l:[0, T] \rightarrow \mathbb{R}$ is a bounded measurable function. Then

$$
z(t) \leq E_{q}\left(\lambda t^{q}\right) z(0)+\int_{0}^{t}(t-s)^{q-1} E_{q, q}\left(\lambda(t-s)^{q}\right) l(s) d s
$$


Proof. Consider the scalar equation

$$
{ }^{C} D_{0}^{q} y(t)=\lambda y(t)+l(t), \quad t \in[0, T],
$$

with the initial condition $y(0)=z(0)$. From inequality (2.9) and equation (2.10), we have

$$
{ }^{C} D_{0}^{q}(z(t)-y(t)) \leq \lambda(z(t)-y(t)) .
$$

There exists a nonnegative function $v:[0, T] \rightarrow \mathbb{R}$ such that

$$
{ }^{C} D_{0}^{q}(z(t)-y(t))=\lambda(z(t)-y(t))-v(t) .
$$

The solution to the last equation is the following nonnegative function

$$
y(t)-z(t)=\int_{0}^{t}(t-s)^{q-1} E_{q, q}\left(\lambda(t-s)^{q}\right) v(s) d s .
$$

Thus, $z(t) \leq y(t)$. Since

$$
y(t)=E_{q}\left(\lambda t^{q}\right) y(0)+\int_{0}^{t}(t-s)^{q-1} E_{q, q}\left(\lambda(t-s)^{q}\right) l(s) d s,
$$

and $y(0)=z(0)$, we finally get the inequality

$$
z(t) \leq E_{q}\left(\lambda t^{q}\right) z(0)+\int_{0}^{t}(t-s)^{q-1} E_{q, q}\left(\lambda(t-s)^{q}\right) l(s) d s .
$$

2.2. Measures of noncompactness and condensing multivalued maps. Let us recall some notions and facts (details can be found, for example, in [24, 25]).

Let $\mathscr{E}$ be a Banach space. We introduce the following notation:

- $\operatorname{Pb}(\mathscr{E})=\{A \subseteq \mathscr{E}: A \neq \varnothing$ is bounded $\}$;

- $P v(\mathscr{E})=\{A \in P b(\mathscr{E}): A$ is convex $\}$

- $K(\mathscr{E})=\{A \in P b(\mathscr{E}): A$ is compact $\}$;

- $K v(\mathscr{E})=P v(\mathscr{E}) \cap K(\mathscr{E})$.

Definition 2.6. Let $(\mathscr{A}, \geq)$ be a nonempty partially ordered set. A function $\beta: P b(\mathscr{E}) \rightarrow \mathscr{A}$ is said to be a measure of noncompactness (MNC) in $\mathscr{E}$ if, for each $\Omega \in \operatorname{Pb}(\mathscr{E})$,

$$
\beta(\overline{\mathrm{co}} \Omega)=\beta(\Omega),
$$

where $\overline{\mathrm{co}} \Omega$ denotes the closure of the convex hull of $\Omega$.

A measure of noncompactness $\beta: P b(\mathscr{E}) \rightarrow \mathscr{A}$ is said to be

1) monotone if $\Omega_{0} \subseteq \Omega_{1}$ implies $\beta\left(\Omega_{0}\right) \leq \beta\left(\Omega_{1}\right)$ for each $\Omega_{0}, \Omega_{1} \in P b(\mathscr{E})$;

2) nonsingular if $\beta(\{a\} \cup \Omega)=\beta(\Omega)$ for each $a \in \mathscr{E}$ and each $\Omega \in P b(\mathscr{E})$.

If $\mathscr{A}$ is the positive cone in an ordered Banach space, the MNC $\beta$ is said to be

4) regular if $\beta(\Omega)=0$ is equivalent to the relative compactness of $\Omega \in P b(\mathscr{E})$;

5) real if $\mathscr{A}$ is the set of all nonnegative real numbers $\mathbb{R}_{+}$with the natural ordering;

6) algebraically semiadditive if $\beta\left(\Omega_{0}+\Omega_{1}\right) \leq \beta\left(\Omega_{0}\right)+\beta\left(\Omega_{1}\right)$ for every $\Omega_{0}, \Omega_{1} \in P b(\mathscr{E})$. 
We mention that the Hausdorff MNC

$$
\chi_{\mathscr{E}}(\Omega)=\inf \{\varepsilon>0: \Omega \text { has a finite } \varepsilon \text {-net }\}
$$

obeys all above properties. Another examples can be presented by the following measures of noncompactness defined on $\operatorname{Pb}(C([0, a] ; E))$, where $C([0, a] ; E)$ is the space of continuous functions with the values in a separable Banach space $E$ :

(i) the modulus of fiber noncompactness

$$
\varphi(\Omega)=\sup _{t \in[0, a]} e^{-p t} \chi_{E}(\Omega(t)),
$$

where $p>0, \chi_{E}$ is the Hausdorff MNC in $E$, and $\Omega(t)=\{y(t): y \in \Omega\}$;

(ii) the modulus of equicontinuity

$$
\bmod _{C}(\Omega)=\lim _{\delta \rightarrow 0} \sup _{y \in \Omega} \max _{\left|t_{1}-t_{2}\right| \leq \delta}\left\|y\left(t_{1}\right)-y\left(t_{2}\right)\right\| .
$$

Notice that these MNCs satisfy all above-mentioned properties except the regularity. For a regular MNC for the space $C([0, a] ; E)$, we can consider

$$
v(\Omega)=\left(\varphi(\Omega), \bmod _{C}(\Omega)\right)
$$

with the values in the positive cone of $\mathbb{R}^{2}$ in the usual partial ordering.

Definition 2.7. Let $X \subseteq \mathscr{E}$ be a closed subset. A multivalued map (or multimap, in short) $\mathscr{F}: X \rightarrow K(\mathscr{E})$ is said to be upper semicontinuous (or u.s.c., in short) if the pre-image

$$
\mathscr{F}^{-1}(V)=\{x \in X: \mathscr{F}(x) \subset V\}
$$

of each open set $V \subset \mathscr{E}$ is open in $X$.

Definition 2.8. An u.s.c. multimap $\mathscr{F}: X \rightarrow K(\mathscr{E})$ is said to be condensing with respect to $a$ MNC $\beta$ (or $\beta$-condensing, in short) if for every bounded set $\Omega \subseteq X$ that is not relatively compact, we have

$$
\beta(\mathscr{F}(\Omega)) \not \beta(\Omega) .
$$

More generally, given a metric space $\Lambda$ of parameters, we say that an u.s.c. multimap $\Gamma$ : $\Lambda \times X \rightarrow K(\mathscr{E})$ is a condensing family with respect to a MNC $\beta$ (or $\beta$-condensing family in short) if for every bounded set $\Omega \subseteq X$ that is not relatively compact, we have

$$
\beta(\Gamma(\Lambda \times \Omega)) \not \beta(\Omega) .
$$

Let $V \subset \mathscr{E}$ be a bounded open set, $\beta$ a monotone nonsingular MNC in $\mathscr{E}$, and $\mathscr{F}: \bar{V} \rightarrow K v(\mathscr{E})$ a $\beta$-condensing multimap such that $x \notin \mathscr{F}(x)$ for all $x \in \partial V$. Here, $\bar{V}$ and $\partial V$ denote the closure and the boundary of the set $V$. In such a setting, the topological degree $\operatorname{deg}(i-\mathscr{F}, \bar{V})$ of the corresponding vector multifield $i-\mathscr{F}$ satisfying standard properties is defined, where $i$ is the identity map on $\mathscr{E}$. In particular,

$$
\operatorname{deg}(i-\mathscr{F}, \bar{V}) \neq 0 \quad \Longrightarrow \quad \text { Fix } \mathscr{F}:=\{x \in V: x \in \mathscr{F}(x)\} \neq \emptyset .
$$

To describe the next property, let us introduce the following notion. 
Definition 2.9. Suppose that two $\beta$-condensing multimaps $\mathscr{F}_{0}, \mathscr{F}_{1}: \bar{V} \rightarrow K v(\mathscr{E})$ have no fixed points on the boundary $\partial V$. The multifields $\Phi_{0}=i-\mathscr{F}_{0}$ and $\Phi_{1}=i-\mathscr{F}_{1}$ are said to be homotopic, and denoted by

$$
\Phi_{0} \sim \Phi_{1}
$$

if there exists a $\beta$-condensing family $\mathfrak{H}:[0,1] \times \bar{V} \rightarrow K(\mathscr{E})$ such that

(i) $x \notin \mathfrak{H}(\lambda, x)$ for all $(\lambda, x) \in[0,1] \times \partial V$;

(ii) $\mathfrak{H}(0, \cdot)=\mathscr{F}_{0}$ and $\mathfrak{H}(1, \cdot)=\mathscr{F}_{1}$.

The homotopy invariance property of the topological degree asserts that if $\Phi_{0} \sim \Phi_{1}$, then $\operatorname{deg}\left(i-\mathscr{F}_{0}, \bar{V}\right)=\operatorname{deg}\left(i-\mathscr{F}_{1}, \bar{V}\right)$.

Let us mention also the following property of the topological degree which we will need in the sequel.

The normalization property. For a constant field $\mathscr{F}(x) \equiv A \in K(\mathscr{E})$, we have

$$
\operatorname{deg}(i-\mathscr{F}, \bar{V})= \begin{cases}1, & \text { if } A \subset V ; \\ 0, & \text { if } A \cap \bar{V}=\emptyset .\end{cases}
$$

\section{MAin RESUlts}

3.1. A priori estimates of solutions. Let $H$ be a separable Hilbert space. We will consider the Cauchy problem for a semilinear fractional order differential inclusion in $H$ :

$$
\begin{gathered}
{ }^{C} D_{0}^{q} x(t) \in A x(t)+F(t, x(t)), \quad t \in[0, T], \\
x(0)=x_{0},
\end{gathered}
$$

where $0<q<1$ and the linear operator $A$ satisfies the following condition:

(A) $A: D(A) \subseteq H \rightarrow H$ is a closed (but not necessarily bounded) linear operator generating a bounded $C_{0}$-semigroup $\left\{U_{A}(t)\right\}_{t \geq 0}$ of linear operators on $H$ and such that

$$
\langle A x, x\rangle \leq-d\|x\|^{2}, \quad \forall x \in D(A)
$$

for some $d>0$.

It will be assumed that the multimap $F:[0, T] \times H \rightarrow K v(H)$ obeys the following conditions:

(F1) the multifunction $F(\cdot, x):[0, T] \rightarrow K v(H)$ admits a measurable selection for each $T>0$ and $x \in H$, i.e., there exists a measurable function $f:[0, T] \rightarrow H$ such that $f(t) \in F(t, x)$ for a.e. $t \in[0, T]$;

(F2) the multimap $F(t, \cdot): H \rightarrow K v(H)$ is u.s.c. for each $T>0$ and a.e. $t \in[0, T]$;

(F3) for each $R>0$ and $T>0$, there exists a function $\omega_{R} \in L^{\infty}[0, T]$ such that

$$
\|F(t, x)\| \leq \omega_{R}(t) \quad \text { for a.e. } t \in[0, T] \text { and for all } x \in H \text { with }\|x\| \leq R ;
$$

(F4) for each $T>0$, there exists $\kappa \in L^{\infty}[0, T]$ such that, for every bounded set $\Omega \subset H$,

$$
\chi(t, \Omega)) \leq \kappa(t) \chi(\Omega),
$$

where $\chi$ denotes the Hausdorff MNC for the space $H$;

(F5) there exists $a \geq 0$ such that

$$
\sup _{y \in F(t, x)}\langle y, x\rangle \leq a\|x\|^{2}+G(t), \quad t \in[0, T]
$$

where $G: \mathbb{R}_{+} \rightarrow \mathbb{R}_{+}$is a locally $L^{\infty}$-function, i.e., $\left.G\right|_{[0, T]} \in L^{\infty}[0, T]$ for each $T>0$. 
From conditions (F1)-(F3), it follows that, for each $T>0$, the superposition multioperator $\mathscr{P}_{F}: C([0, T] ; H) \multimap L^{\infty}((0, T) ; H)$ is defined by the formula

$$
\mathscr{P}_{F}(x)=\left\{f \in L^{\infty}((0, T) ; H): f(s) \in F(s, x(s)) \text { for a.e. } s \in[0, T]\right\}
$$

(see, for example, $[24,25])$.

Let us recall (see, for example, $[5,8-10]$ ) that a mild solution to problem (3.1), (3.2) is a function $x \in C([0, T], H)$ of the form

$$
x(t)=\mathscr{G}_{A}(t) x_{0}+\int_{0}^{t}(t-s)^{q-1} \mathscr{T}_{A}(t-s) f(s) d s,
$$

where

$$
\mathscr{G}_{A}(t)=\int_{0}^{\infty} \xi_{q}(\theta) U_{A}\left(t^{q} \theta\right) d \theta, \quad \mathscr{T}_{A}(t)=q \int_{0}^{\infty} \theta \xi_{q}(\theta) U_{A}\left(t^{q} \theta\right) d \theta,
$$

$f \in \mathscr{P}_{F}(x)$ and the function $\xi_{q}$ is defined by formula (2.3).

Lemma 3.1 (See [14, Lemma 3.4]). The operator functions $\mathscr{G}_{A}$ and $\mathscr{T}_{A}$ possess the following properties:

1) for each $t \in[0, T]$, the operator functions $\mathscr{G}_{A}(t)$ and $\mathscr{T}_{A}(t)$ are bounded linear operators. More precisely, for each $x \in H$,

$$
\begin{gathered}
\left\|\mathscr{G}_{A}(t) x\right\|_{H} \leq M\|x\|_{H}, \\
\left\|\mathscr{T}_{A}(t) x\right\|_{H} \leq \frac{q M}{\Gamma(1+q)}\|x\|_{H},
\end{gathered}
$$

where

$$
M=\sup _{t \in[0,+\infty)}\left\|U_{A}(t)\right\|
$$

2) the operator functions $\mathscr{G}_{A}(\cdot)$ and $\mathscr{T}_{A}(\cdot)$ are strongly continuous, i.e., the functions $t \in$ $[0, T] \rightarrow \mathscr{G}_{A}(t) x$ and $t \in[0, T] \rightarrow \mathscr{T}_{A}(t) x$ are continuous for each $x \in H$.

Remark 3.2. Notice that if $A$ is a bounded linear operator, then the solution defined by formula (3.4) satisfies the following differential equation (see [22])

$$
{ }^{C} D_{0}^{q} x(t)=A x(t)+f(t) .
$$

Now, suppose that $x \in C([0, T] ; H)$ is any mild solution to problem (3.1)-(3.2). Take a measurable selection $f \in \mathscr{P}_{F}(x)$ satisfying (3.4). Then condition (F3) implies that

$$
\|f(t)\| \leq \omega_{R}(t) \text { for a.e. } t \in[0, T],
$$

where

$$
R=\|x\|_{C([0, T] ; H)} \quad \text { and } \quad \omega_{R} \in L^{\infty}(0, T) .
$$

The following assertion holds true.

Lemma 3.3. For each $\varepsilon>0$, there exists a set $m_{\varepsilon} \subset[0, T]$ with Lebesgue measure $\mu\left(m_{\varepsilon}\right)<\varepsilon$, and a piecewise linear function $g_{\varepsilon}:[0, T] \rightarrow H$ with a finite number of nodes belonging to $D(A)$ such that

$$
\left\|f(t)-g_{\varepsilon}(t)\right\|<\varepsilon, \quad t \in[0, T] \backslash m_{\varepsilon} .
$$


Proof. Consider the functions $f_{\gamma}:[0, T] \rightarrow H$ defined by the formula

$$
f_{\gamma}(t)=\frac{1}{2 \gamma} \int_{t-\gamma}^{t+\gamma} \bar{f}(s) d s
$$

where

$$
\bar{f}(s)= \begin{cases}f(s), & \text { for } s \in[0, T], \\ 0, & \text { for } s \notin[0, T] .\end{cases}
$$

Then $f_{\gamma}(t) \rightarrow f(t)$ for a.e. $t \in[0, T]$ as $\gamma \rightarrow 0^{+}$, since, for a measurable function, the Lebesgue points form a complete measure space (see [26]). Notice that the functions $f_{\gamma}$ are continuous and

$$
\left\|f_{\gamma}(t)\right\| \leq\|f\|_{L^{\infty}([0, T], H)} \text { for } t \in[0, T] .
$$

Hence each function $f_{\gamma}$ may be approximated with an arbitrary degree of accuracy in the space $C([0, T] ; H)$ by piecewise linear functions $g_{\delta}$ with a finite number of nodes belonging to $D(A)$.

Take a sequence $\gamma_{k} \rightarrow 0^{+}$. Applying to functions $f_{\gamma_{k}}$ the Egorov theorem (see [27]), for a given $\varepsilon>0$, we may find $m_{\varepsilon} \subset[0, T]$ such that $\mu\left(m_{\varepsilon}\right)<\varepsilon$ and the sequence $\left\{f_{\gamma_{k}}\right\}$ uniformly converges to $f$ on $[0, T] \backslash m_{\varepsilon}$. So, we have, for a sufficiently large $k$

$$
\left\|f(t)-f_{\gamma_{k}}(t)\right\|<\frac{\varepsilon}{2} \text { for } t \in[0, T] \backslash m_{\varepsilon} .
$$

Taking now a piecewise linear function $g_{\varepsilon}$ satisfying

$$
\left\|f_{\gamma_{k}}-g_{\varepsilon}\right\|_{C([0, T], H)}<\frac{\varepsilon}{2}
$$

we get the desired assertion immediately.

Now, taking a piecewise linear function $g_{\varepsilon}$ satisfying conditions of Lemma 3.3, we consider the function

$$
x^{\varepsilon}(t)=\mathscr{G}_{A}(t) x_{0}^{\varepsilon}+\int_{0}^{t}(t-s)^{q-1} \mathscr{T}_{A}(t-s) g_{\varepsilon}(s) d s
$$

where $x_{0}^{\varepsilon} \in D(A)$ and $x_{0}^{\varepsilon} \rightarrow x_{0}$ as $\varepsilon \rightarrow 0^{+}$.

Lemma 3.4. The expression

$$
I(\varepsilon)(t):=\int_{0}^{t}(t-s)^{q-1}\left\|\mathscr{T}_{A}(t-s)\right\| \cdot\left\|g_{\varepsilon}(s)-f(s)\right\| d s
$$

tends to zero as $\varepsilon \rightarrow 0^{+}$uniformly on $[0, T]$.

Proof. Denoting

we get from (3.6) the operator norms

$$
N=\frac{q M}{\Gamma(1+q)}
$$

$$
\left\|\mathscr{T}_{A}(t)\right\| \leq N, \quad t \in[0, T] .
$$

For a given $\gamma>0$, we choose $\sigma>0$ such that

$$
\frac{\sigma^{q}}{q} N\left(2\left\|\omega_{R}\right\|_{L^{\infty}}+1\right)<\frac{\gamma}{2}
$$

From the construction of the function $g_{\varepsilon}$ (see relations (3.10) and (3.11)), it follows that, for a sufficiently small $\varepsilon>0$,

$$
\left\|g_{\mathcal{E}}(t)\right\| \leq\left\|\omega_{R}\right\|_{L^{\infty}}+1, \quad t \in[0, T] .
$$


Then, for the case $t \leq \sigma \leq T$, we have

$$
\begin{aligned}
\int_{0}^{t}(t-s)^{q-1}\left\|\mathscr{T}_{A}(t-s)\right\| \cdot\left\|g_{\varepsilon}(s)-f(s)\right\| d s & \leq \int_{0}^{\sigma}(t-s)^{q-1} N\left(\left\|g_{\varepsilon}(s)\right\|+\|f(s)\|\right) d s \\
& \leq N\left(2\left\|\omega_{R}\right\|_{L^{\infty}}+1\right) \int_{0}^{\sigma}(t-s)^{q-1} d s \\
& =\frac{\sigma^{q}}{q} N\left(2\left\|\omega_{R}\right\|_{L^{\infty}}+1\right) \\
& <\frac{\gamma}{2} .
\end{aligned}
$$

If $\sigma<t$, then

$$
\begin{aligned}
I(\varepsilon)(t)= & \int_{t-\sigma}^{t}(t-s)^{q-1}\left\|\mathscr{T}_{A}(t-s)\right\| \cdot\left\|g_{\varepsilon}(s)-f(s)\right\| d s \\
& +\int_{0}^{t-\sigma}(t-s)^{q-1}\left\|\mathscr{T}_{A}(t-s)\right\| \cdot\left\|g_{\varepsilon}(s)-f(s)\right\| d s \\
= & I_{1}(\varepsilon)(t)+I_{2}(\varepsilon)(t) .
\end{aligned}
$$

For $I_{1}(\varepsilon)$, the following estimate holds:

$$
I_{1}(\varepsilon)(t) \leq \frac{\sigma^{q}}{q} N\left(2\left\|\omega_{R}\right\|_{L^{\infty}}+1\right)<\frac{\gamma}{2}
$$

For $I_{2}(\varepsilon)$, we have

$$
\begin{aligned}
I_{2}(\varepsilon)(t)= & \int_{[0, t-\sigma] \backslash m_{\mathcal{\varepsilon}}}(t-s)^{q-1}\left\|\mathscr{T}_{A}(t-s)\right\| \cdot\left\|g_{\mathcal{\varepsilon}}(s)-f(s)\right\| d s \\
& +\int_{[0, t-\sigma] \cap m_{\varepsilon}}(t-s)^{q-1}\left\|\mathscr{T}_{A}(t-s)\right\| \cdot\left\|g_{\mathcal{\varepsilon}}(s)-f(s)\right\| d s \\
= & I_{21}(\varepsilon)(t)+I_{22}(\varepsilon)(t) .
\end{aligned}
$$

Set

$$
N_{1}=\max _{s \in[0, t-\sigma]}(t-s)^{q-1}\left\|\mathscr{T}_{A}(t-s)\right\| .
$$

Since $\left\|g_{\varepsilon}(s)-f(s)\right\|<\varepsilon$ for $s \in[0, t-\sigma] \backslash m_{\varepsilon}$, we obtain the estimate

$$
I_{21}(\varepsilon)(t) \leq N_{1} \varepsilon(t-\sigma)<N_{1} \varepsilon T .
$$

For $I_{22}(\varepsilon)$, we have

$$
I_{22}(\varepsilon)(t) \leq N_{1}\left(2\left\|\omega_{R}\right\|_{L^{\infty}}+1\right) \mu\left([0, t-\sigma] \cap m_{\varepsilon}\right) \leq N_{1}\left(2\left\|\omega_{R}\right\|_{L^{\infty}}+1\right) \varepsilon .
$$

Choosing $\varepsilon>0$ so that

$$
N_{1}\left(T+2\left\|\omega_{R}\right\|_{L^{\infty}}+1\right) \varepsilon<\frac{\gamma}{2}
$$

we get the desired conclusion immediately.

Corollary 3.5. The expression $\left\|x^{\varepsilon}-x\right\|_{C([0, T] ; H)}$ tends to zero as $\varepsilon \rightarrow 0^{+}$.

Proof. We have the estimation

$$
\left\|x(t)-x^{\varepsilon}(t)\right\| \leq\left\|\mathscr{G}_{A}(t)\left(x_{0}-x_{0}^{\varepsilon}\right)\right\|+\int_{0}^{t}(t-s)^{q-1}\left\|\mathscr{T}_{A}(t-s)\right\| \cdot\left\|g_{\varepsilon}(s)-f(s)\right\| d s .
$$


Since the operator function $\mathscr{G}_{A}(t)$ is strongly continuous and $x_{0}^{\varepsilon} \rightarrow x_{0}$ as $\varepsilon \rightarrow 0^{+}$, we have that the first term in this sum tends to zero uniformly on $[0, T]$. The second term tends to zero uniformly due to Lemma 3.4.

Remark 3.6. In Lemma 3.4, we can replace the term $\left\|\mathscr{T}_{A}(t-s)\right\|$ with the Mittag-Leffler function $E_{q, q}\left((-d+a) t^{q}\right)$. Repeating the above arguments, we get

$$
\int_{0}^{t}(t-s)^{q-1} E_{q, q}\left((-d+a)(t-s)^{q}\right) \cdot\left\|g_{\varepsilon}(s)-f(s)\right\| d s \rightarrow 0
$$

uniformly on $[0, T]$ as $\varepsilon \rightarrow 0^{+}$.

Consider the Yosida approximations for the operator $A$ :

$$
A_{n}=n A(n I-A)^{-1}, \quad n \geq 1 .
$$

It is known (see, e.g., $[27,28])$ that $A_{n}$ are bounded and mutually commuting linear operators. Moreover, $A_{n}$ converges to $A$ pointwise on $D(A)$, and each $A_{n}$ generates a uniformly continuous contraction semigroup $U_{A_{n}}$. Introduce the approximations $x_{n}^{\varepsilon}$ by the formulas

$$
x_{n}^{\varepsilon}(t)=\mathscr{G}_{A_{n}}(t) x_{0}^{\varepsilon}+\int_{0}^{t}(t-s)^{q-1} \mathscr{T}_{A_{n}}(t-s) g_{\varepsilon}(s) d s,
$$

Lemma 3.7. For a fixed $\varepsilon>0$, the sequence $x_{n}^{\varepsilon}$ converges to $x^{\varepsilon}$ uniformly on $[0, T]$ as $n \rightarrow \infty$.

Proof. For each fixed $x \in H$, we have

$$
U_{A_{n}}(t) x \rightarrow U_{A}(t) x
$$

uniformly with respect to $t \in[0, T]$ (see [28]). Consequently, we also have the uniform convergence

$$
\mathscr{G}_{A_{n}}(t) x \rightarrow \mathscr{G}_{A}(t) x \text { and } \mathscr{T}_{A_{n}}(t) x \rightarrow \mathscr{T}_{A}(t) x,
$$

that implies for a fixed $\varepsilon>0$ the desired convergence.

Due to the closedness of the operator $A$, we have, for $x \in D(A)$,

$$
A U_{A}(t) x=U_{A}(t) A x, \quad A A_{n} x=A_{n} A x, \quad \text { and } \quad A U_{A_{n}}(t) x=U_{A_{n}}(t) A x .
$$

By the definition of the operator functions $\mathscr{G}_{A}(t)$ and $\mathscr{T}_{A}(t)$, for $x \in D(A)$, we have

$$
\begin{gathered}
A \mathscr{G}_{A}(t) x=\mathscr{G}_{A}(t) A x, \quad A \mathscr{T}_{A}(t) x=\mathscr{T}_{A}(t) A x, \\
A \mathscr{G}_{A_{n}}(t) x=\mathscr{G}_{A_{n}}(t) A x, \quad \text { and } \quad A \mathscr{T}_{A_{n}}(t) x=\mathscr{T}_{A_{n}}(t) A x .
\end{gathered}
$$

Since for a given piecewise linear function $g_{\varepsilon}$ the set $\left\{A g_{\varepsilon}(s): s \in[0, T]\right\}$ is compact in $H$, it follows that the range of the function $g_{\varepsilon}$ lies in $D(A)$. Therefore, $\left\{A x_{n}^{\varepsilon}(t): t \in[0, T]\right\}$ is a compact set.

Lemma 3.8. For a fixed $\varepsilon>0$, we have

$$
\left(n(n I-A)^{-1}-I\right) A x_{n}^{\varepsilon}(t) \rightarrow 0
$$

as $n \rightarrow \infty$ uniformly with respect to $t \in[0, T]$. 
Proof. Since $\left(n(n I-A)^{-1}-I\right) y \rightarrow 0$ for each fixed $y \in H$ (see [28]), we have

$$
\left(n(n I-A)^{-1}-I\right) A x \rightarrow 0
$$

uniformly with respect to $x \in\left\{g_{\varepsilon}(t): t \in[0, T]\right\}$. Since $x_{n}^{\varepsilon}$ can be expressed through $g_{\varepsilon}$ by the formula (3.13), we get the desired assertion immediately.

Now, we are in a position to present the main result of this section.

Theorem 3.9. Assume that Conditions $(A)$ and $(F 1)-(F 5)$ hold. Then there exists a continuous function $\mathscr{C}:[0,+\infty) \rightarrow[0,+\infty)$ such that, for every mild solution x of problem (3.1)-(3.2) defined on an interval $[0, T]$, the following priori estimate holds true:

$$
\|x\|_{C([0, T], H)} \leq \mathscr{C}(T) .
$$

Proof. Take a sequence of positive numbers $\theta_{k} \rightarrow 0$, and choose a sequence of approximations $\left\{x^{\varepsilon_{k}}\right\}$ by (3.12) so that

$$
\left\|x^{\varepsilon_{k}}-x\right\|_{C([0, T], H)}<\theta_{k} .
$$

Further, according to Corollary 3.5, we find $n^{1}\left(\varepsilon_{k}\right)$ such that, for $n \geq n^{1}\left(\varepsilon_{k}\right)$,

$$
\left\|x_{n}^{\varepsilon_{k}}-x^{\varepsilon_{k}}\right\|_{C([0, T], H)}<\theta_{k} .
$$

Since $x_{n}^{\varepsilon}$ lies in $D(A)$ and the sequence $\left\{A x_{n}^{\varepsilon}\right\}$ for each fixed $\varepsilon>0$ is uniformly bounded in $n$ (see Lemma 3.8), we find that there is $n^{2}\left(\varepsilon_{k}\right)$ such that, for $n \geq n^{2}\left(\varepsilon_{k}\right)$,

$$
\sup _{t \in[0, T]}\left\langle\left(n(n I-A)^{-1}-I\right) A x_{n}^{\varepsilon}(t), x_{n}^{\varepsilon}(t)\right\rangle<\theta_{k} .
$$

Take $n_{k}=\max \left(n^{1}\left(\varepsilon_{k}\right), n^{2}\left(\varepsilon_{k}\right)\right)$. It follows that

$$
\begin{gathered}
\left\|x_{n_{k}}^{\varepsilon_{k}}-x^{\varepsilon_{k}}\right\|_{C([0, T], H)}<\theta_{k}, \\
\sup _{t \in[0, T]}\left\langle\left(n_{k}\left(n_{k} I+A\right)^{-1}-I\right) A x_{n_{k}}^{\varepsilon_{k}}(t), x_{n_{k}}^{\varepsilon_{k}}(t)\right\rangle<\theta_{k} .
\end{gathered}
$$

Notice that simultaneously we construct the corresponding sequences of functions $\left\{g_{\varepsilon_{k}}\right\}$ and sets $m_{\varepsilon_{k}}$ (see Lemma 3.3). By virtue of Remark 3.2, we have

$$
{ }^{C} D_{0}^{q} x_{n_{k}}^{\varepsilon_{k}}(t)=A_{n_{k}} x_{n_{k}}^{\varepsilon_{k}}(t)+g_{\varepsilon_{k}}(t)
$$

From $[16,17]$, it follows that

$$
{ }^{C} D_{0}^{q}\left\|x_{n_{k}}^{\varepsilon_{k}}(t)\right\|^{2} \leq\left\langle A_{n_{k}} x_{n_{k}}^{\varepsilon_{k}}(t), x_{n_{k}}^{\varepsilon_{k}}(t)\right\rangle+\left\langle g_{\varepsilon_{k}}(t), x_{n_{k}}^{\varepsilon_{k}}(t)\right\rangle .
$$

Now let us estimate the right hand side of inequality (3.17). Using Conditions (A) and (F1)(F5), and setting $R=\|x\|_{C([0, T] ; H)}$, we have

$$
\begin{aligned}
\left\langle A_{n_{k}} x_{n_{k}}^{\varepsilon_{k}}(t), x_{n_{k}}^{\varepsilon_{k}}(t)\right\rangle+\left\langle g_{\varepsilon_{k}}(t), x_{n_{k}}^{\varepsilon_{k}}(t)\right\rangle \\
=\left\langle A x_{n_{k}}^{\varepsilon_{k}}(t), x_{n_{k}}^{\varepsilon_{k}}(t)\right\rangle+\left\langle\left(n_{k}\left(n_{k} I-A\right)^{-1}-I\right) A x_{n_{k}}^{\varepsilon_{k}}(t), x_{n_{k}}^{\varepsilon_{k}}(t)\right\rangle \\
\quad+\left\langle g_{\varepsilon_{k}}(t)-f(t), x_{n_{k}}^{\varepsilon_{k}}(t)\right\rangle+\langle f(t), x(t)\rangle+\left\langle f(t), x_{n_{k}}^{\varepsilon_{k}}(t)-x(t)\right\rangle \\
\leq-d\left\|x_{n_{k}}^{\varepsilon_{k}}(t)\right\|^{2}+a\|x(t)\|^{2}+\left\langle g_{\varepsilon_{k}}(t)-f(t), x_{n_{k}}^{\varepsilon_{k}}(t)\right\rangle+\left\langle f(t), x_{n_{k}}^{\varepsilon_{k}}(t)-x(t)\right\rangle \\
\quad+\left\langle\left(n_{k}\left(n_{k} I-A\right)^{-1}-I\right) A x_{n_{k}}^{\varepsilon_{k}}(t), x_{n_{k}}^{\varepsilon_{k}}(t)\right\rangle+G(t) \\
\leq(-d+a)\left\|x_{n_{k}}^{\varepsilon_{k}}(t)\right\|^{2}+a\left\|x(t)-x_{n_{k}}^{\varepsilon_{k}}(t)\right\|\left(\|x(t)\|+\left\|x_{n_{k}}^{\varepsilon_{k}}(t)\right\|\right)+\left\|g_{\varepsilon_{k}}(t)-f(t)\right\|\left\|x_{n_{k}}^{\varepsilon_{k}}(t)\right\| \\
\quad+\|f(t)\|\left\|x_{n_{k}}^{\varepsilon_{k}}(t)-x(t)\right\|+\left\langle\left(n_{k}\left(n_{k} I-A\right)^{-1}-I\right) A x_{n_{k}}^{\varepsilon_{k}}(t), x_{n_{k}}^{\varepsilon_{k}}(t)\right\rangle+G(t) .
\end{aligned}
$$


For sufficiently large $k$, we get the inequality

$$
\begin{aligned}
{ }^{C} D_{0}^{q}\left\|x_{n_{k}}^{\varepsilon_{k}}(t)\right\|^{2} \leq & (-d+a)\left\|x_{n_{k}}^{\varepsilon_{k}}(t)\right\|^{2}+3 a R \theta_{k}+\theta_{k}\|f(t)\|+\theta_{k} \\
& +2 R\left\|g_{\varepsilon_{k}}(t)-f(t)\right\|+G(t) .
\end{aligned}
$$

By virtue of Lemma 2.5, we find that the following inequality holds true

$$
\begin{aligned}
\left\|x_{n_{k}}^{\varepsilon_{k}}(t)\right\|^{2} \leq & E_{q}\left((-d+a) t^{q}\right)\left\|x_{0}^{\varepsilon_{k}}\right\|^{2}+\int_{0}^{t}(t-s)^{q-1} E_{q, q}\left((-d+a)(t-s)^{q}\right) G(s) d s \\
& +\theta_{k} \int_{0}^{t}(t-s)^{q-1} E_{q, q}\left((-d+a)(t-s)^{q}\right)\left(3 a R+\omega_{R}(s)+1\right) d s \\
& +2 R \int_{0}^{t}(t-s)^{q-1} E_{q, q}\left((-d+a)(t-s)^{q}\right)\left\|g_{\varepsilon_{k}}(s)-f(s)\right\| d s .
\end{aligned}
$$

Notice that the third and forth terms tends to zero as $k \rightarrow \infty$. In fact, in the third term, the integral is uniformly bounded on $[0, T]$, and we can apply Remark 3.6 to the forth term. Letting $k \rightarrow \infty$ in (3.18) yields that

$$
\|x(t)\|^{2} \leq E_{q}\left((-d+a) t^{q}\right)\left\|x_{0}\right\|+\int_{0}^{t}(t-s)^{q-1} E_{q, q}\left((-d+a)(t-s)^{q}\right) G(s) d s
$$

Therefore, the right hand side determines the function of a priori estimate $\mathscr{C}$ on the interval $[0, T]$. This completes the proof.

3.2. Existence result. From Theorem 3.9, we can obtain the following result on the existence of a solution to problem (3.1)-(3.2) on an arbitrary interval $[0, T]$.

Theorem 3.10. Assume that Conditions (A) and (F1)-(F5) hold. Then problem (3.1)-(3.2) has a mild solution on $[0, T]$ for each $T>0$.

Proof. Consider the family of multivalued integral operators

$$
\mathfrak{F}: C([0, T] ; H) \times[0,1] \multimap C([0, T] ; H)
$$

defined in the following way:

$$
\mathfrak{F}(x, \lambda)=\left\{z=\mathscr{G}_{A}(t) x_{0}+\lambda \int_{0}^{t}(t-s)^{q-1} \mathscr{T}_{A}(t-s) f(s) d s: f \in \mathscr{P}_{F}(x)\right\},
$$

where $\mathscr{P}_{F}$ is the superposition multioperator defined by (3.3). It is clear that each fixed point $x_{\lambda} \in C([0, T] ; H)$ of the multimap $\mathfrak{F}(\cdot, \lambda), \lambda \in[0,1]$ is a mild solution to the problem

$$
\begin{gathered}
{ }^{C} D_{0}^{q} x(t) \in A x(t)+\lambda F(t, x(t)), \quad t \in[0, T], \\
x(0)=x_{0} .
\end{gathered}
$$

It is known (see [5, 6, 8-10]) that the family (3.20) has compact convex values and is condensing with respect to the MNC $v$ in $C([0, T] ; H)$ (see Section 2). It also holds that the multioperators satisfy conditions (F1)-(F5) independently on $\lambda$. By Theorem 3.9, we conclude that there exists a constant $\mathscr{C}(T)$ such that all solutions to problem (3.21)-(3.22) satisfy the priori estimate

$$
\left\|x_{\lambda}\right\| \leq \mathscr{C}(T) .
$$

Therefore, the multioperators $\mathfrak{F}(\cdot, \lambda)$ from family (3.20) are fixed point free on the boundary of the ball $\mathfrak{B}$ of the space $C([0, T] ; H)$ centered at zero and of the radius $\mathscr{C}(T)+1$. 
Notice that the range of the multioperator $\mathfrak{F}(\cdot, 0)$ consists of the single function $y(t)=\mathscr{G}_{A}(t) x_{0}$ being its fixed point. Applying the homotopy and the normalization properties of the topological degree, we obtain

$$
\operatorname{deg}(i-\mathfrak{F}(\cdot, 1), \mathfrak{B})=\operatorname{deg}(i-\mathfrak{F}(\cdot, 0), \mathfrak{B})=1 .
$$

This yields, by the existence property of the topological degree, the desired result.

3.3. Bounded solutions on the semi-axis. The priori estimate (3.19) helps us to verify the following assertion about the existence of bounded solutions on $[0, \infty)$ to problem (3.1)-(3.2).

Theorem 3.11. Suppose that $d>a$, conditions $(A)$ and $(F 1)-(F 5)$ hold true, and the function $G$ in condition (F5) belongs to the space $L^{r}[0, \infty)$, where $r>\frac{1}{q}$. Then, each mild solution to problem (3.1)- (3.2) is bounded on $[0, \infty)$.

Proof. By virtue of Theorem 3.9, all mild solutions to problem (3.1)-(3.2) are defined on [0, $)$ and satisfy estimate (3.19). Since

$$
E_{q}\left((-d+a) t^{q}\right)\left\|x_{0}\right\| \rightarrow 0 \quad \text { as } \quad t \rightarrow \infty
$$

to prove the theorem, it is sufficient to demonstrate that the expression

$$
\int_{0}^{t}(t-s)^{q-1} E_{q, q}\left((-d+a)(t-s)^{q}\right) G(s) d s
$$

is bounded while $t \in(0, \infty)$.

For $\tau>T$, let (see [19])

$$
0 \leq E_{q, q}((-d+a) \tau)<\frac{c}{\tau},
$$

where $c$ is a constant. Then, for $\tau>T^{\frac{1}{q}}$,

$$
0 \leq E_{q, q}\left((-d+a) \tau^{q}\right) \mid<\frac{c}{\tau^{q}}
$$

For $\tau \leq T^{\frac{1}{q}}$, we have the estimate $E_{q, q}\left(\tau^{q}\right) \leq M$. Estimate, for large $t$, expression (3.23):

$$
\begin{aligned}
& \left|\int_{0}^{t}(t-s)^{q-1} E_{q, q}\left((-d+a)(t-s)^{q}\right) G(s) d s\right| \\
& \leq \int_{t-T^{\frac{1}{q}}}^{t}(t-s)^{q-1} E_{q, q}\left((-d+a)(t-s)^{q}\right) G(s) d s \\
& \quad+\int_{0}^{t-T^{\frac{1}{q}}}(t-s)^{q-1} E_{q, q}\left((-d+a)(t-s)^{q}\right) G(s) d s=I_{1}(t)+I_{2}(t) .
\end{aligned}
$$

Let $\frac{1}{p}+\frac{1}{r}=1$. By the Holder inequality,

$$
I_{1}(t) \leq\left(\int_{t-T^{\frac{1}{q}}}^{t}(t-s)^{p(q-1)} M^{p} d s\right)^{\frac{1}{p}}\left(\int_{t-T^{\frac{1}{q}}}^{t} G^{r}(s) d s\right)^{\frac{1}{r}} .
$$

From the fact that $r>\frac{1}{q}$ implies the inequality

$$
p(q-1)+1>1
$$

we get

$$
I_{1}(t) \leq\left(\frac{T^{\frac{p(q-1)+1}{q}}}{p(q-1)+1}\right)^{\frac{1}{p}}\|G\|_{L^{r}[0, \infty)}
$$


For $I_{2}$, we have the estimate

$$
\begin{aligned}
I_{2}(t) & \leq \int_{T^{\frac{1}{q}}}^{t} \tau^{q-1} \frac{c}{\tau^{q}} G(t-\tau) d \tau \\
& \leq c\left(\int_{T^{\frac{1}{q}}}^{t} \tau^{-p} d \tau\right)^{\frac{1}{p}}\left(\int_{T^{\frac{1}{q}}}^{t} G(t-\tau) d \tau\right)^{\frac{1}{r}} \\
& \leq c\left(\frac{T^{\frac{1}{q}(1-p)}}{p-1}\right)^{\frac{1}{p}}\|G\|_{L^{r}[0, \infty)} .
\end{aligned}
$$

This completes the proof.

\section{Acknowledgments}

The authors are grateful to the reviewers for useful suggestions which improved the contents of this paper. N.-C. Wong expresses his deep appreciation to the colleagues in Tiangong University for their warm hospitality during his visit there. The work was supported by the State contract of the Russian Ministry of Science and Higher Education as part of the state task (contract FZGF-2020-0009).

\section{REFERENCES}

[1] E.A. Coddington, N. Levinson, Theory of Ordinary Differential Equations, McGraw-Hill Book Company, Inc., New York, Toronto, London, 1955.

[2] P. Hartman, Ordinary Differential Equations, John Wiley and Sons, New York, London, Sydney, 1964.

[3] M.A. Krasnoselskii, The Operator of Translation along the Trajectories of Differential Equations. Translation of Mathematical Monographs, 19, American Mathematical Society, Providence, RI, 1968.

[4] M. Afanasova, Y.C. Liou, V. Obukhoskii, G. Petrosyan, On controllability for a system governed by a fractional-order semilinear functional differential inclusion in a Banach space, J. Nonlinear Convex Anal. 20 (2019), 1919-1935.

[5] M. Afanasova, G. Petrosyan, On the boundary value problem for functional differential inclusion of fractional order with general initial condition in a Banach space, Russian Math. 63 (2019), 1-12.

[6] I. Benedetti, V. Obukhovskii, V. Taddei, On generalized boundary value problems for a class of fractional differential inclusions, Fract. Calc. Appl. Anal. 20 (2017), 1424-1446.

[7] F. Mainardi, On the initial value problem for the fractional diffusion-wave equation, in Waves and Stability in Continuous Media, S. Rionero and T. Ruggeri (eds.), pp. 246-251, World Scientific, Singapore, 1994

[8] M. Kamenskii, V. Obukhovskii, G. Petrosyan, J.C. Yao, On semilinear fractional order differential inclusions in Banach spaces, Fixed Point Theory. 18 (2017), 269-292.

[9] M. Kamenskii, V. Obukhovskii, G. Petrosyan, J.C. Yao, On approximate solutions for a class of semilinear fractional-order differential equations in Banach spaces, Fixed Point Theory Appl. 28 (2017), 1-28.

[10] M. Kamenskii, V. Obukhovskii, G. Petrosyan, J.C. Yao, Existence and Approximation of Solutions to Nonlocal Boundary Value Problems for Fractional Differential Inclusions, Fixed Point Theory Appl. 2019 (2019), 2 .

[11] T.D. Ke, N.V. Loi, V. Obukhovskii, Decay solutions for a class of fractional differential variational inequalities, Fract. Calc. Appl. Anal. 18 (2015), 531-553.

[12] T.D. Ke, V. Obukhovskii, N.C. Wong, J.C. Yao, On a class of fractional order differential inclusions with infinite delays, Appl. Anal. 92 (2013), 115-137.

[13] G.G. Petrosyan, M.S. Afanasova, On the Cauchy problem for differential inclusion of fractional order with a nonlinear boundary condition, Bulletin of Voronezh State University. Series Physics. Maths. 1 (2017), 135151. (In Russian)

[14] Z. Zhang, B. Liu, Existence of mild solutions for fractional evolution equations, Fixed Point Theory 15 (2014), 325-334. 
[15] Y. Zhou, F. Jiao, Existence of mild solutions for fractional neutral evolution equations, Comput. Math. Appl. 59 (2010), 1063-1077.

[16] N. Aguila-Camacho, M. A. Duarte-Mermoud, J. A. Gallegos, Lyapunov functions for fractional order systems, Commun. Nonlinear Sci.d Numer. Simul. 19 (2014), 2951-2957

[17] M.I. Gomoyunov, Fractional derivatives of convex Lyapunov functions and control problems in fractional order systems, Fractional Calculus Appl. Anal. 21 (2018), 1238-1261.

[18] M.I. Gomoyunov, Approximation of fractional order conflict-controlled systems, Progress in Fractional Differentiation and Appl. 5 (2019), 143-155.

[19] R. Gorenflo, A.A. Kilbas, F. Mainardi, S.V. Rogosin, Mittag-Leffler Functions, Related Topics and Applications, Springer-Verlag, Berlin Heidelberg, 2014.

[20] A.A. Kilbas, H.M. Srivastava, J.J. Trujillo, Theory and Applications of Fractional Differential Equations, Elsevier Science B.V., North-Holland Mathematics Studies, Amsterdam, 2006.

[21] I. Podlubny, Fractional Differential Equations, Academic Press, San Diego, 1999.

[22] Y. Zhou, Fractional Evolution Equations and Inclusions: Analysis and Control, Elsevier Academic Press, London, 2016.

[23] R.-N. Wang, D.-H. Chen, T.-J. Xiao, Abstract fractional Cauchy problems with almost sectorial operators, J. Differential Equations. 252 (2012), 202-235.

[24] M. Kamenskii, V. Obukhovskii, P. Zecca, Condensing Multivalued Maps and Semilinear Differential Inclusions in Banach Spaces, Walter de Gruyter, Berlin-New-York, 2001.

[25] V. Obukhovskii, B. Gel'man, Multivalued Maps and Differential Inclusions. Elements of Theory and Applications, World Scientific Publishing Co., Hackensack, NJ, 2020.

[26] V. M. Bogdan, Generalized vectorial Lebesgue and Bochner integration theory, arXiv:1006.3881v1 [math.FA]. 2010.

[27] K. Yosida, Functional Analysis, Springer-Verlag, Berlin, Heidelberg, New York, 1980.

[28] K.-J. Engel, R. Nagel, A Short Course on Operator Semigroups, Springer-Verlag, New York, 2006. 